ARTICLE

\title{
LRF maintains genome integrity by regulating the non-homologous end joining pathway of DNA repair
}

Xue-Song Liu ${ }^{1, \star, \dagger}$, Gurushankar Chandramouly ${ }^{2, \dagger}$, Emilie Rass ${ }^{2}$, Yinghua Guan ${ }^{3}$, Guocan Wang ${ }^{1}$, Robin M. Hobbs ${ }^{1}$, Anbazhagan Rajendran ${ }^{2}$, Anyong Xie ${ }^{2, \dagger}$, Jagesh V. Shah ${ }^{3}$, Anthony J. Davis ${ }^{4}$, Ralph Scully ${ }^{2}$, Andrea Lunardi ${ }^{1, \star, \dagger}$ \& Pier Paolo Pandolfi ${ }^{1}$

Leukemia/lymphoma-related factor (LRF) is a POZ/BTB and Krüppel (POK) transcriptional repressor characterized by context-dependent key roles in cell fate decision and tumorigenesis. Here we demonstrate an unexpected transcription-independent function for LRF in the classical non-homologous end joining (CNHEJ) pathway of double-strand break (DSB) repair. We find that LRF loss in cell lines and mouse tissues results in defective cNHEJ, genomic instability and hypersensitivity to ionizing radiation. Mechanistically, we show that LRF binds and stabilizes DNA-PKCs on DSBs, in turn favouring DNA-PK activity. Importantly, LRF loss restores ionizing radiation sensitivity to p53 null cells, making LRF an attractive biomarker to direct p53-null LRF-deficient tumours towards therapeutic treatments based on genotoxic agents or PARP inhibitors following a synthetic lethal strategy.

\footnotetext{
${ }^{1}$ Cancer Research Institute, Beth Israel Deaconess Cancer Center, Department of Medicine and Pathology, Beth Israel Deaconess Medical Center, Harvard Medical School, Boston, Massachusetts 02215, USA. ${ }^{2}$ Cancer Research Institute, Beth Israel Deaconess Cancer Center, Department of Medicine, Beth Israel Deaconess Medical Centre, Harvard Medical School, Boston, Massachusetts 02215, USA. ${ }^{3}$ Department of Systems Biology, Harvard Medical School, 4 Blackfan Circle, HIM 564, Boston, MA 02115, USA. ${ }^{4}$ Division of Molecular Radiation Biology, Department of Radiation Oncology, University of Texas Southwestern Medical Centre, 2201 Inwood Rd, Dallas, Texas 75390, USA. * These authors contributed equally to this work. † Present address: School of Life Science and Technology, ShanghaiTech University, Shanghai 201203, China (X.-S.L.); Fels Institute for Cancer Research and Molecular Biology, Temple University, 3500N. Broad Street, Philadelphia, Pennsylvania 19140, USA (G.C.); Sir Run Run Shaw Hospital and Institute of Translational Medicine, Zhejiang University School of Medicine, Hangzhou, Zhejiang 310016, China (A.X.); Centre for Integrative Biology (CIBIO), University of Trento, 38123 Trento, Italy (A.L.). Correspondence and requests for materials should be addressed to R.S. (email: rscully@bidmc.harvard.edu) or to A.L.(email: andrea.lunardi@unitn.it) or to P.P.P. (email: ppandolf@bidmc.harvard.edu).
} 
T he ability to maintain a stable genome is crucial for normal cell function, and genomic instability may underlie many developmental disorders and human diseases, including cancer $^{1}$. DNA double-strand breaks (DSBs) are perhaps the most deleterious threat to genomic stability. Cells use two main pathways to repair DSBs: non-homologous end joining (NHEJ) and homologous recombination $(\mathrm{HR})^{2}$. These two pathways are largely distinct from one another. HR is particularly effective in S and G2 phases when the break is repaired using genetic information from a sister chromatid, whereas NHEJ can be effective at all times in the cell cycle, yet it is often error prone $e^{3}$. The DNA-dependent protein kinase (DNA-PK) complex, including catalytic subunit DNA-PKcs and DNA-binding subunits Ku70/80, is a key component of the classical non-homologous end joining (cNHEJ) apparatus. The physical interaction between DNA-bound $\mathrm{Ku}(\mathrm{Ku} 70 / \mathrm{Ku} 80)$, in particular the C-terminal tail of Ku80, and DNA-PKcs at sites of DNA breaks defines a functional DNA-PK complex that concomitantly bridges the broken DNA ends and activates the DNA repair machinery through the phosphorylation of specific downstream targets ${ }^{4,5}$.

LRF (formerly known as POKEMON ${ }^{6}$, FBI-1 (ref. 7) or $\mathrm{OCZF}^{8}$ ) is encoded by the ZBTB7A gene, and is a member of the POZ/BTB and Krüppel (POK) family of transcription factors. POK transcription factors can bind DNA through a Krüppellike-DNA-binding domain and repress transcription by recruiting co-repressor complexes through the POZ (Pox virus and Zinc finger) domain ${ }^{9}$. POK transcription factors have been recognized as critical developmental regulators and have been directly implicated in human cancer ${ }^{10}$. For example, BCL6 (B-Cell Lymphoma 6) and PLZF (Promyelocytic Leukemia Zinc Finger) are critical players in the pathogenesis of Non-Hodgkin's Lymphoma and acute promyelocytic leukemia, respectively ${ }^{11,12}$. LRF shares structural similarities with BCL6 and PLZF and plays critical context-dependent role in embryonic development, haematopoiesis and tumorigenesis ${ }^{6,13-19}$.

In this work, we identify a novel and transcriptional independent function for LRF in the maintenance of genomic stability by regulation of cNHEJ. Mechanistically, we demonstrate that LRF is rapidly recruited on the sites of DNA damage where, by binding DNA-PKcs, it stabilizes the DNA-PK complex, in turn promoting DNA-PKcs kinase activity and efficient DSB repair. Importantly, LRF downregulation, a frequent hallmark of different types of human cancer, restores radiation sensitivity in p53 null cells, thus becoming a new potential biomarker of remarkable therapeutic relevance.

\section{Results}

LRF is required for maintenance of genomic integrity. LRF is a critical repressor of the tumour suppressor gene Arf, and cells such as mouse embryonic fibroblasts (MEFs), which lack Lrf become refractory to oncogenic transformation and undergo premature senescence ${ }^{6}$. In an effort to identify new functions of LRF unrelated to Arf regulation through a clean genetic approach, we compared the effects of acute Lrf deletion in Lrffloxflox or Arf ${ }^{-1-}$ Lrflox/flox MEFs through infection with a Cre recombinase-containing retrovirus. Although Cre expression in both wild-type and $\mathrm{Arf}^{-/-}$MEFs had no effect on cell proliferation (Supplementary Fig. 1a), and Cre-mediated deletion of Lrf in Lrflox/flox MEFs triggered the expected growth suppression through Arf-dependent cellular senescence ${ }^{6}$ (Fig. 1a), surprisingly, loss of Lrf caused a profound growth suppression in the $A r f^{-1-}$ MEFs as well (Fig. 1a). The growth defect of $A r f^{-1-} \operatorname{Lrf}$ deleted $\left(\mathrm{Arf}^{-1-} \operatorname{Lrfff} \mathrm{cre}\right)$ MEFs was accompanied by evidence of chromosome breakage, as shown by Giemsa staining of metaphase chromosome spreads (Fig. 1b). Telomere Fish fluorescent in situ hybridization staining of chromosome spreads also indicated accumulation of chromosome breaks, aneuploidy, polyploidy and abnormal chromosomes in $\mathrm{Arf}^{-1-}$ Lrf deleted MEFs (Supplementary Fig. 1b). Accordingly, neutral comet assay showed a significant accumulation of DNA DSBs in $\operatorname{Lrf}$ deleted MEFs (Fig. 1c), and immunofluorescence and western blot studies confirmed a marked increase in $\gamma$-H2AX staining (Fig. 1d,e). To further characterize this phenotype, we assessed whether LRF conditional inactivation triggers unrepaired DNA damage in vivo. Villin-Cre and Mx1-Cre transgenes were used to delete floxed Lrf in the mouse intestine and hematopoietic systems, respectively ${ }^{20,21}$. Importantly, in LRF conditional knockout intestine and spleen the downregulation of LRF (Supplementary Fig. 1c) was associated with a significant increase of $\gamma-\mathrm{H} 2 \mathrm{AX}$ levels (Fig. 1f), suggestive of persistent DNA damage in these cells ${ }^{22}$.

LRF deficiency sensitizes cells to ionizing radiation. Since LRF inactivation results in persistent DNA damage and genomic instability, we used clonogenic survival assays to assess the sensitivity of $A r f^{-/-}$and $A r f^{-/-}$Lrf deleted MEFs to different types of DNA-damaging agents. These included $\gamma$-radiation, the radiomimetic drug phleomycin, the Topoisomerase II inhibitor ICRF-193, the Topoisomerase I inhibitor Camptothecin, and the DNA cross-linking agent, mitomycin C. Compared with $\mathrm{Arf}^{-1-}$ control MEFs, Arf $^{-{ }^{-}}$Lrf deleted cells revealed hypersensitivity to $\gamma$-radiation, phleomycin and ICRF-193 (Fig. 2a,b and Supplementary Fig. 2a), but no alteration in mitomycin C and Camptothecin sensitivity (Fig. 2c, and Supplementary Fig. 2b). Furthermore, upon treatment with phleomycin at various concentrations, $A r f^{-/-} \operatorname{Lrf}$ deleted MEFs displayed a significant increase of comet tail DNA content and $\gamma$-H2AX levels (Fig. 2d,e). We then further tested in vivo whether Lrf null mutants are hypersensitive to ionizing radiation (IR). Constitutive $L r f$ inactivation results in embryonic lethality ${ }^{6}$, while conditional Lrf inactivation in the adult hematopoietic system (' $\operatorname{Lrf} \mathrm{cKO}$ '), using Mx1-Cre and $\mathrm{pIpC}$ induction, is compatible with a normal lifespan ${ }^{14}$. After a single dose of whole-body $\gamma$-irradiation $(7.5 \mathrm{~Gy})$, all $\operatorname{Lrf} c K O$ mice died within 16 days, while all wild-type control mice remained healthy for 2 weeks after irradiation (Fig. 2f). After irradiation, $\operatorname{Lrf} c \mathrm{KO}$ bone marrow cells accumulated much more unrepaired DNA damage (shown by $\gamma$-H2AX staining) and became apoptotic (by cleaved caspase 3 staining). Lrf cKO mice were found to have died from acute bone marrow failure (Supplementary Fig. 2c).

LRF participates in Xrcc4-dependent NHEJ. To directly test whether a specific DSB repair process requires LRF function, we next took advantage of selective DSB repair reporter assays (Fig. 3a, and Supplementary Fig. 3c) ${ }^{23}$. Notably, siRNA-mediated knockdown of LRF caused a significant decrease in NHEJ repair efficiency as shown by decreased I-SceI-induced GFP expression in NHEJ reporter cells (Fig. 3b). Classical NHEJ (cNHEJ) is a rapid and efficient process, requiring DNA Ligase IV and XRCC4. In cells lacking either of these genes, rejoining of DSBs occurs through a slower, highly error-prone process termed 'alternative end joining' $(\mathrm{aEJ})^{4,5,24-26}$. Using biallelically deleted $X r c c 4$ (here termed $\operatorname{Xrcc}^{\Delta / \Delta}$ ) NHEJ reporter ES cells, we observed only a modest and statistically insignificant decrease of NHEJ efficiency after Lrf knockdown (Fig. 3c). Similarly, pharmacological inhibition of DNA-PKcs activity (NU7441) combined with siRNA-mediated knockdown of LRF showed only a mild and statistically insignificant reduction of NHEJ efficiency in Xrcc4 proficient ES cells when compared 
a

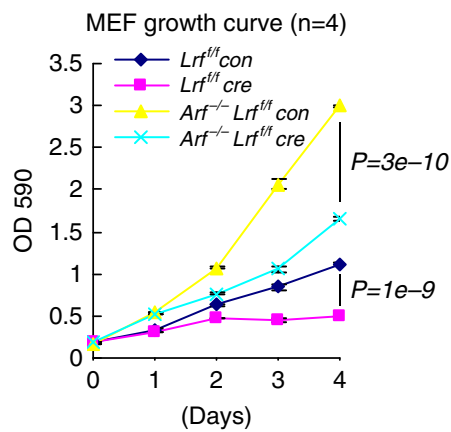

d

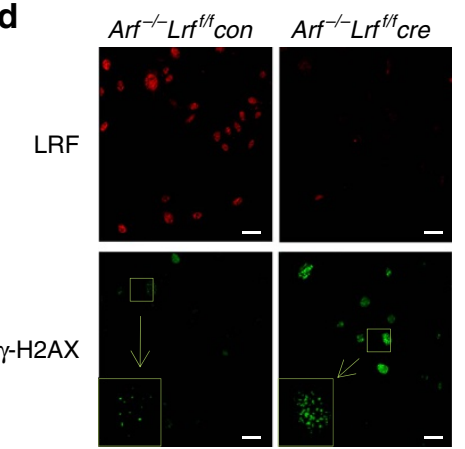

e

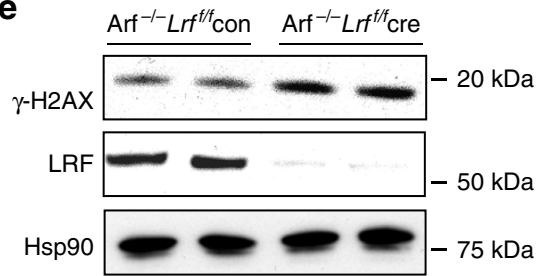

b

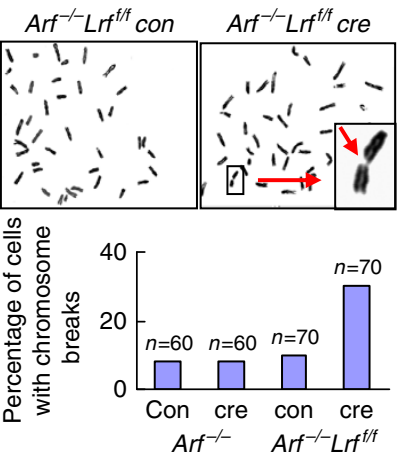

C

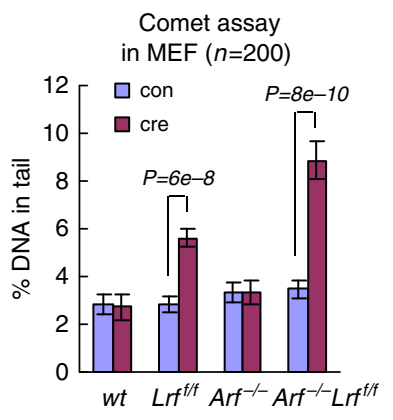

$\mathbf{f}$
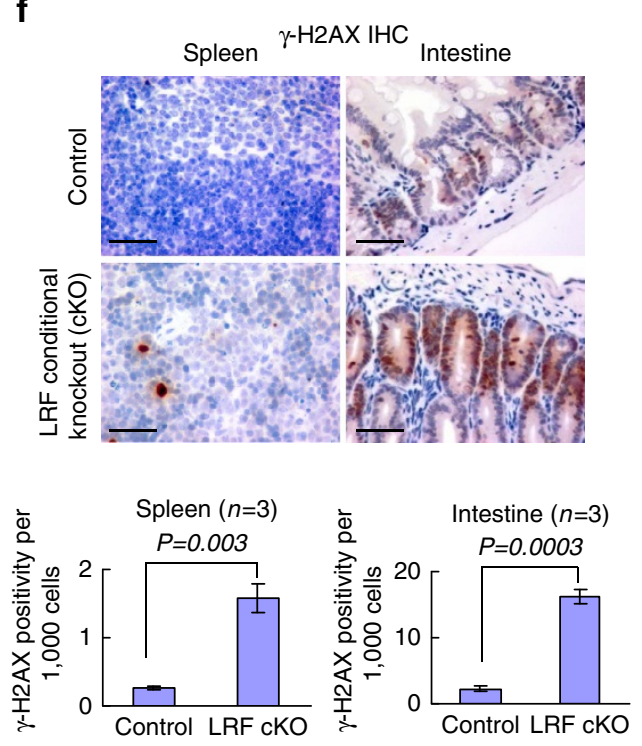

Figure 1 | LRF is required for maintenance of genome integrity. (a) Growth curve of control and Lrf deleted MEFs in both wild-type and Arf null backgrounds. Data from $n=4$ independent experiments are presented as mean \pm s.e.m. Associated $P$ value calculated by Student's $t$-test analysis is indicated. (b) Metaphase chromosome spreads stained with Giemsa. Insert is an enlargement of a typical chromosome break. Ratio of cells with chromosome breaks was counted in $\mathrm{Arf}^{-/-}$and $\mathrm{Arf}^{-/-} \mathrm{Lrf}^{\mathrm{f} / \mathrm{f}} \mathrm{MEFs}$ transduced with Control or Cre expression retrovirus. (c) Detection of DSBs by neutral comet assay, in wild-type, $\mathrm{Lrf}^{f / f}, \mathrm{Arf}^{-/}-$and $\mathrm{Arf}^{-/}-\mathrm{Lrf} / / \mathrm{f}$ MEFs transduced with control or Cre expression retrovirus. The percentage of DNA in comet tails is scored from 200 cells of 3 different experiments and presented as mean \pm s.e.m. Associated $P$ value calculated by Student's $t$-test analysis is indicated. (d,e) Increased $\gamma-\mathrm{H} 2 \mathrm{AX}$ in Arf $^{-/}-$Lrf deleted MEFs as shown by immunofluorescence (scale bar, $\left.10 \mu \mathrm{m}\right)(\mathbf{d})$ and immunoblot (e). Hsp90 is used as a loading control for the immunoblot. (f) Paraffin sections of $\mathrm{rrf}^{+/ /+}$Villin-Cre (control) or $\mathrm{Lrf}^{f / f}$ Villin-Cre C57/BL6 mouse intestine (3 months old), $\mathrm{Lrf}^{+/+} \mathrm{Mx1}$-Cre (control) or $\mathrm{Lrf} / \mathrm{f} \mathrm{Mx1}-\mathrm{Cre}$ C57/BL6 mouse spleen (4 weeks after four PIPC injection) were immunostained with $\gamma$-H2AX antibody (scale bar, $100 \mu \mathrm{m}$ ). The fractions of $\gamma-\mathrm{H} 2 \mathrm{AX}$ positive cells in control and Lrf conditional knockout spleen and intestine were scored. Data from $n=3$ different mice are presented as mean \pm s.e.m. Associated $P$ value calculated by Student's $t$-test analysis is indicated. Scale bar, $100 \mu \mathrm{m}$.

with DNA-PKcs inhibition alone (Supplementary Fig. 3a) ${ }^{23}$. On the other hand, depletion of LRF had no impact on rejoining of I-SceI-induced DSBs in human U2OS cells carrying a reporter of micro-homology-mediated end joining ${ }^{27}$ (Supplementary Fig. 3b), a frequent mediator of $a E J^{27,28}$. We found that LRF is also dispensable for $\mathrm{HR}$, based on experiments performed using mouse ES and U2OS cells carrying an HR reporter ${ }^{29}$ (Fig. 3d and Supplementary Fig. 3c,d).

Collectively, these results clearly define LRF as a novel important player in Xrcc4/DNA-PK-dependent cNHEJ pathway of DSB repair. In future experiments, it will be instructive to define more fully the structure-function analysis of LRFDNA-PKcs interaction as well as the epistatic relationships between LRF inactivation and the loss of other c-NHEJ genes.

LRF is known to act as a transcription factor. We therefore used microarray analysis in wild-type and $\operatorname{Lrf}$ conditional knockout MEFs to decrypt its activity in DSB repair
(Supplementary Tables 1 and 2). Surprisingly, Lrf knockout cells did not display significant alterations in the expression of genes known to be essential for cNHEJ either at early (Supplementary Table 2) or at late passages (Supplementary Fig 3e,f). Only the expression of MRE11, implicated in both aEJ and cNHEJ, resulted downregulated in Lrf null cells compared with wild-type (Supplementary Tables 1 and 2). Although MRE11 mild downregulation could explain the slight reduction in the efficiency of aEJ noted in Fig. 3c, the much more pronounced impact on the cNHEJ pathway in LRF-depleted cells (Fig. 3b) suggests a more fundamental role of LRF in this mechanism of DSB repair. Strongly supporting this hypothesis, chromatin immunoprecipitation (ChIP) experiments demonstrated the binding of LRF to site-specific DSBs generated by I-SceI (Fig. 3e), while an in vivo imaging approach proved the ability of LRF to localize to the vicinity of DSBs generated by laser damage (Fig. 3f, upper panel), with a kinetic closely comparable 

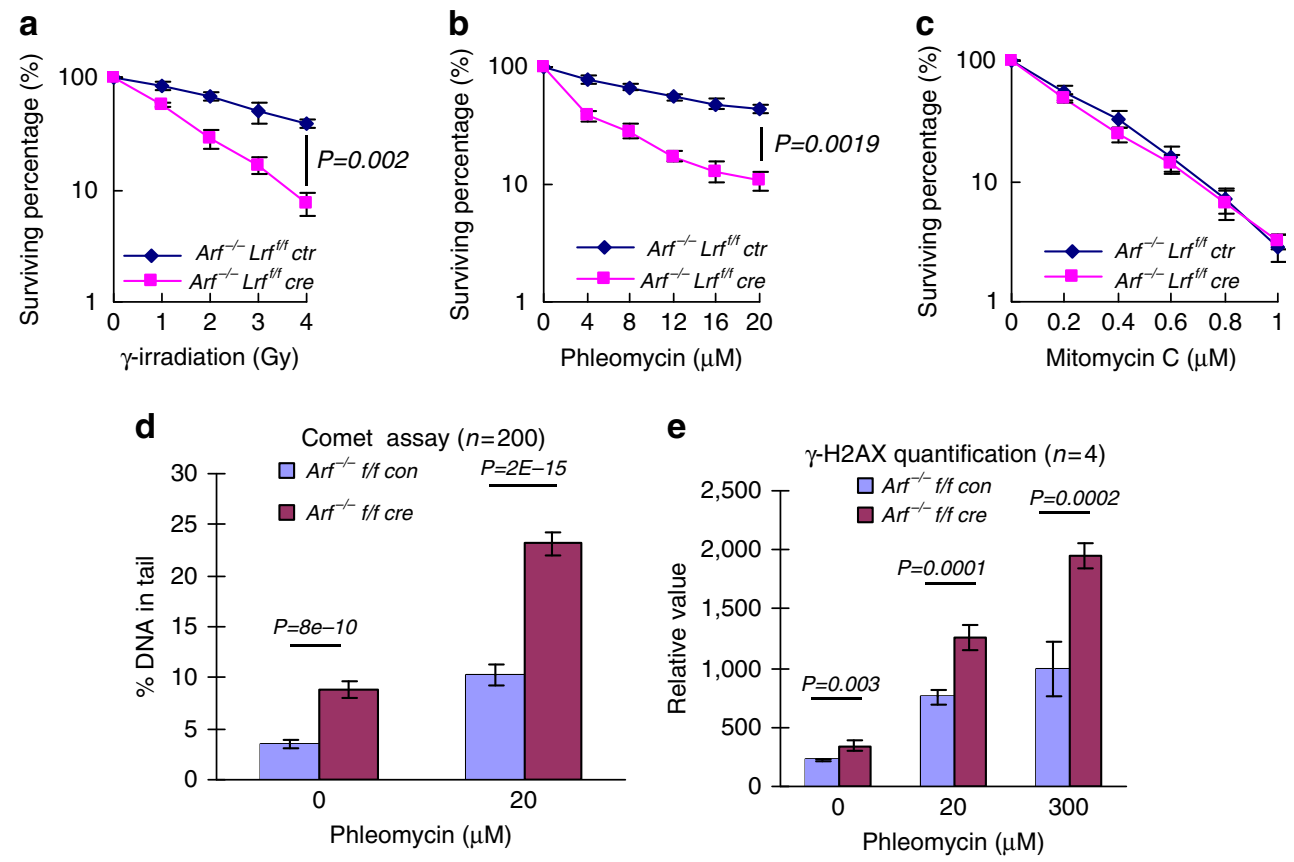

f

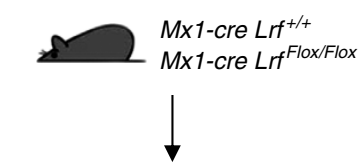

8 Weeks old, 5XplpC injection
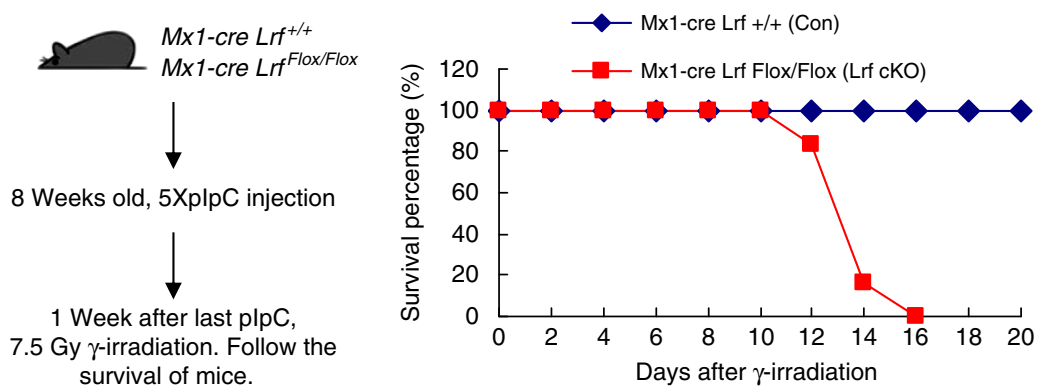

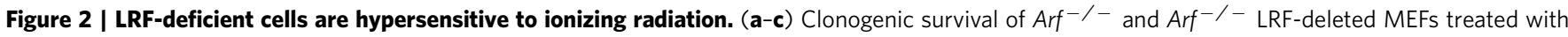
$\gamma$-radiation (a), phleomycin (b) and mitomycin C (c). Data from $n=4$ independent experiments are presented as mean \pm s.e.m. Associated $P$ value calculated by Student's $t$-test analysis is indicated. (d) DNA damage levels in Arf ${ }^{-/}-$and Arf ${ }^{-/-}$LRF-deleted MEF treated with phleomycin assessed by comet assay. The percentage of DNA in comet tails is scored from 200 cells of three different experiments and presented as mean \pm s.e.m. Associated $P$ value calculated by Student's $t$-test analysis is indicated. (e) $\gamma-\mathrm{H} 2 \mathrm{AX}$ levels assessed by flow cytometric analysis of Arf $^{-} /{ }^{-}$and Arf ${ }^{-} /-$LRF deleted $^{-}$ MEFs, $1 \mathrm{~h}$ after 20 or $300 \mu \mathrm{M}$ phleomycin treatment. Data from $n=4$ independent experiments are presented as mean $\pm \mathrm{s}$.e.m. Associated $P$ value calculated by Student's $t$-test analysis is indicated. (f) Survival curve of Lrf hematopoietic system conditional knockout mice ( $L r f$ cKO) ( $n=6$ ) and sibling control mice $(n=9)$ after single dose of whole-body $\gamma$-radiation (7.5 Gy).

to other cNHEJ proteins, such that of Ku80 and DNA-PKcs ${ }^{30}$. Importantly, LRF recruitment to DSBs is not dependent on DNA-PKcs or Ku80 (Fig. 3f, lower panels-middle/right).

Taken together, these results point to a transcriptional independent role for LRF in cNHEJ, a conclusion consistent with the observation that $L r f$-deleted cells are hypersensitive to IR, phleomycin and ICRF-193, but not to mitomycin C and camptothecin.

LRF interacts with DNA-PKcs and regulates DNA-PK function. To determine the transcriptional independent role of LRF in cNHEJ, we first assessed whether LRF could associate with DSB repair protein complexes. To this end, LRF-associated proteins were isolated through tandem affinity purification from HeLa cells stably expressing human LRF tagged with Flag and haemagglutinin (HA) epitopes and analysed by mass spectrometry. Importantly, we found LRF associated with the DNA-PK protein complex, including DNA-PKcs, Ku70 and Ku80 (Supplementary Fig. 4a). Mass spectrometry data were then validated in pull-down experiments with overexpressed FLAG/ HA tagged LRF (Supplementary Fig. 4b, and Supplementary Table 3), as well as through the reciprocal co-immunoprecipitation of endogenous LRF with DNA-PKcs, Ku70 or Ku80 (Fig. 4a). The association between DNA-PKcs and the Ku70/Ku80 heterodimer is DNA dependent ${ }^{31}$. We therefore determined whether the association between LRF and DNA-PKcs or Ku requires DNA. Endogenous co-immunoprecipitations in the presence of ethidium bromide $\left(50 \mu \mathrm{g} \mathrm{ml}^{-1}\right)$, which disrupts DNA-dependent interactions, indicated that the association between LRF and $\mathrm{Ku}$ is strictly dependent on the presence of DNA, while the interaction between LRF and DNA-PKcs, although favoured by DNA, persists in its absence (Fig. 4b). Furthermore, in vitro binding with FLAG-tagged LRF and purified DNA-PK components also indicated that the binding of LRF to Ku70 and Ku80 requires DNA, while DNA bridging is not necessary for the interaction between DNA-PKcs and LRF (Fig. 4c). Interestingly, we observed that, in the absence of Lrf, the mobilization of DNA-PKcs to the chromatin fraction following DNA damage, is significantly decreased (Fig. $4 \mathrm{~d}$, and 
a

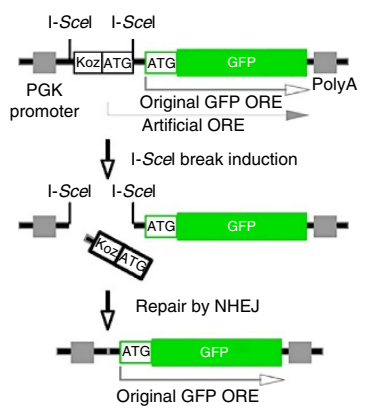

b

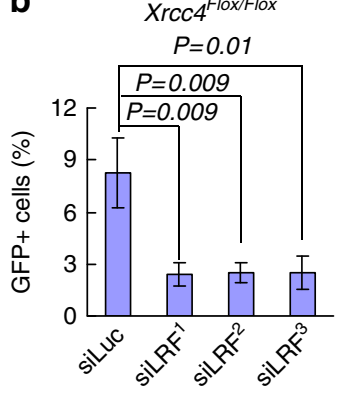

c

C $\quad \operatorname{Xrcc}^{\Delta / \Delta}$

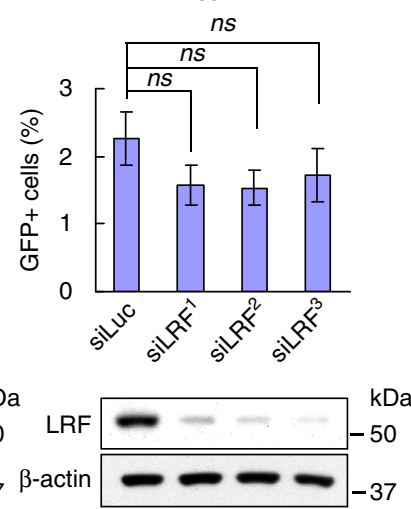

d

e
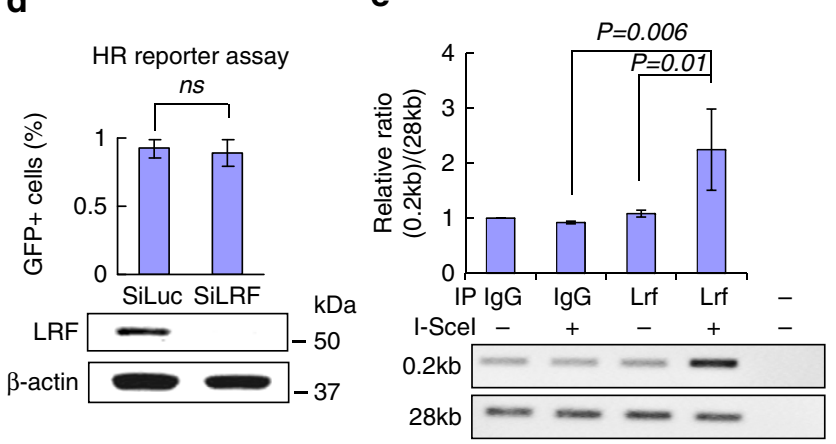

f
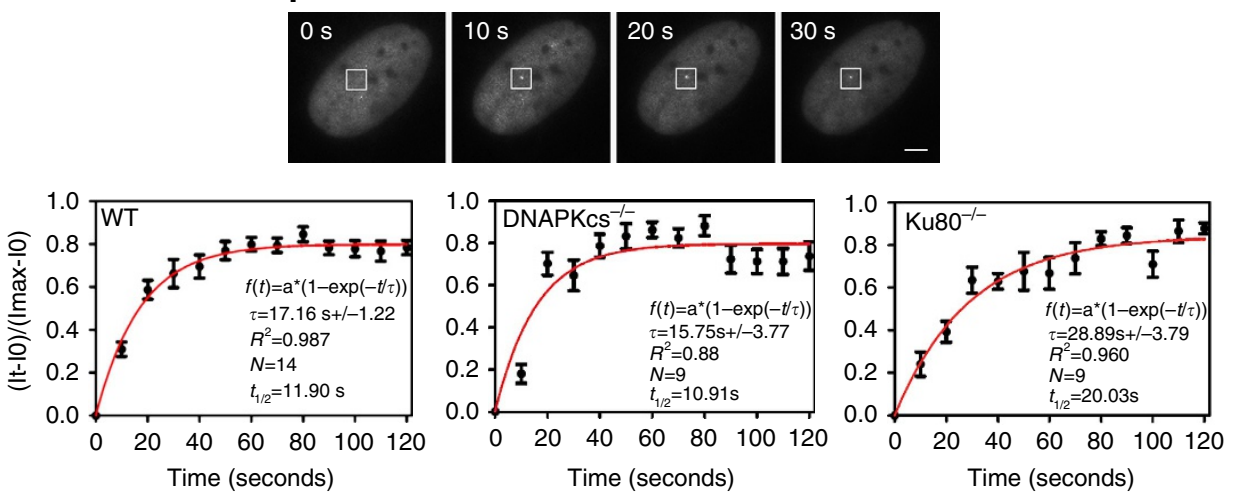

Figure 3 | Transcriptional independent role of LRF in cNHEJ. (a) Structure of the NHEJ reporter. 'Koz-ATG' denotes an artificial Kozak-ATG translation

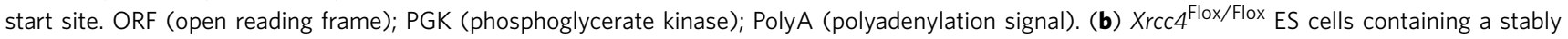
integrated NHEJ reporter were subject to LRF knockdown using three independent siRNAs. Statistically significant reductions in GFP-positive cells are shown for each LRF siRNA. Data from four independent experiments are presented as mean values \pm s.e.m. Associated $P$ value calculated by Student's $t$-test analysis is indicated. (c) Effect of LRF siRNAs on NHEJ in isogenic Xrcc $4^{\Delta / \Delta}$ NHEJ reporter ES cells. Differences in the amount of GFP-positive siLuc and siLRF transfected cells are not statistically significant. Data from four independent experiments are presented as mean \pm s.e.m. Associated $P$ value calculated by Student's $t$-test analysis is indicated. (d) Effect of LRF siRNA on homologous recombination efficiency using a specific reporter assay (see also Supplementary Fig. 3c). Differences in the amount of GFP-positive siLuc and siLRF transfected cells are not statistically significant. Data from 4 independent experiments are presented as mean values \pm s.e.m. Associated $P$ value calculated by Student's $t$-test analysis is indicated. (e) Recruitment of Lrf to site-specific DNA breaks generated by I-Scel. ChIP analysis indicates the significant recruitment of LRF to DNA breaks induced by I-Scel. Average values of $n=3$ independent experiments are shown as mean values \pm s.d. Associated $P$ value calculated by Student's $t$-test analysis is indicated. (f) GFP-LRF recruitment to DSB sites generated by a multiphoton laser system. LRF kinetics of recruitment to DSBs were evaluated in wild-type, DNA-PKcs ${ }^{-/-}$and $\mathrm{Ku} 80^{-/-}$cells. Average values of $n=20$ independent acquisitions are shown as mean values \pm s.d. Scale bar, $1 \mu \mathrm{m}$

Supplementary Fig. 4c). Furthermore, significantly less DNA-PKcs was co-immunoprecipitated with $\mathrm{Ku}$ antibodies in LRF-depleted cells compared with controls (Fig. $4 \mathrm{e}$ and Supplementary Fig. 4d,e). Defects in the formation and stabilization of the Ku/DNA-PKcs/DNA complex may predict impaired DNA-PKcs kinetics in response to DNA damage. To test this hypothesis, we expressed YFP-tagged DNA-PKcs or $\mathrm{Ku} 80$ constructs in control and $\operatorname{sh} L R F$ stable U2OS cells. Strikingly, in a context where the kinetics of recruitment/release of Ku80 were not affected by LRF (Supplementary Fig. 4f), we observed that the retention time, but not the recruitment time, of DNA-PKcs on the laser-induced breaks was significantly 
a

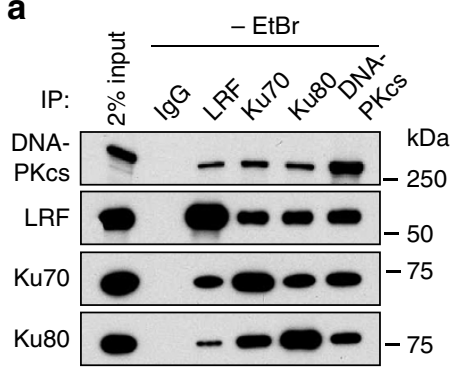

b

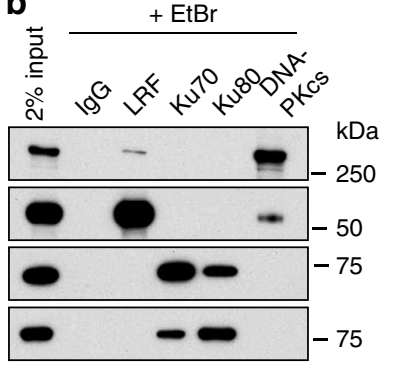

c

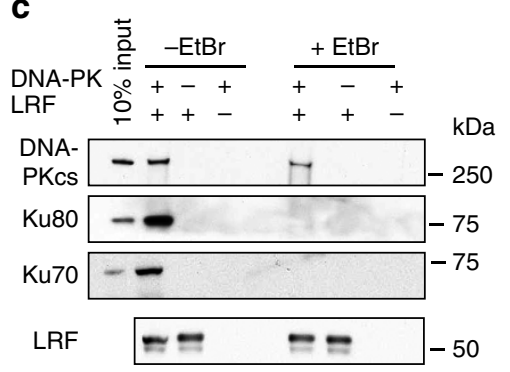

d

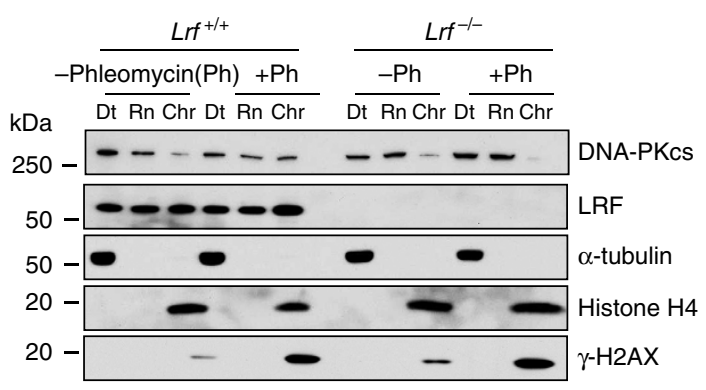

e

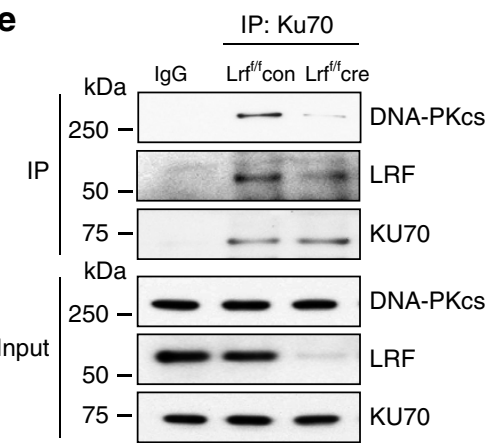

f
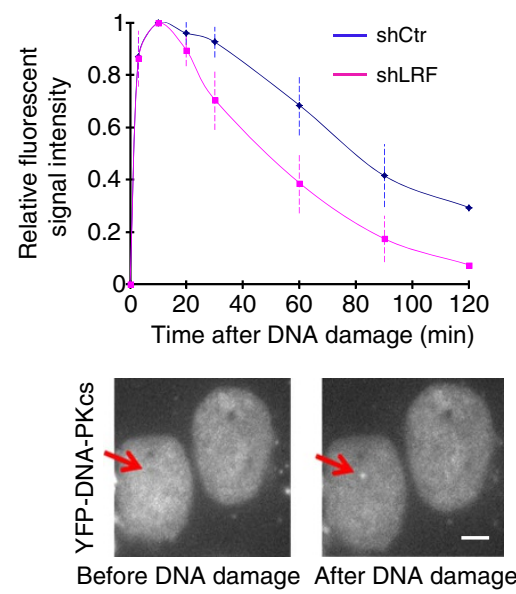

g

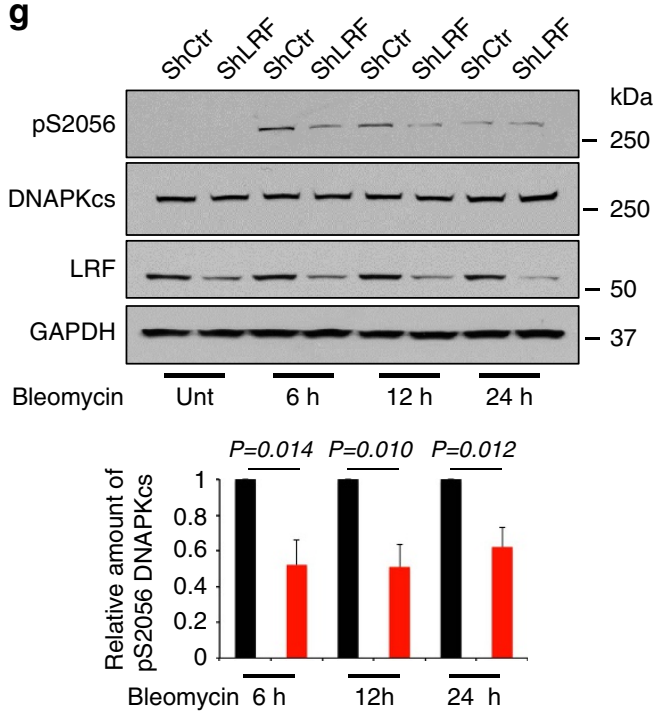

Figure 4 | Interaction of LRF with DNA-PKcs and Ku70/80. (a,b) Endogenous interaction between LRF and DNA-PKcs/Ku70/Ku80 in the absence (a) or presence (b) of ethidium bromide (EtBr) $\left(50 \mu \mathrm{g} \mathrm{ml}{ }^{-1}\right.$ ). (c) In vitro LRF and DNA-PK binding assay. FLAG-tagged LRF was purified by immunoprecipitation with Flag antibody affinity resin then washed with high salt buffer $(500 \mathrm{mM} \mathrm{NaCl})$. Purified DNA-PK components were added in the absence or presence of EtBr and the association of Ku70, Ku80 or DNA-PKcs with LRF was assessed by western blotting. (d) LRF stabilizes DNA-PKcs on chromatin in response to DNA damage. $p 53^{-/-} \mathrm{Lrf}^{+/+}$and $p 53^{-/-} \mathrm{Lrf}^{-/-}$cells were fractionated into detergent extractable (Dt), RNase extractable (Rn) and RNase-resistant chromatin (Chr) compartments. (e) Ku70 co-immunoprecipitation performed in control ( $p 53^{-/-} \mathrm{Lrf} / / f \mathrm{ctr}$ ) and LRF conditional knockout ( $p 53^{-/-}$Lrf//f cre) MEF. (f) YFP tagged DNA-PKcs was expressed in stable shCtr and shLRF U2OS cells. Association and dissociation kinetics of YFP-DNA-PKcs recruitment to DNA damage foci are shown. Average values of 20 cells are presented as mean values $\pm \mathrm{s}$.d. Scale bar, $1 \mu \mathrm{m}$. (g) Relative amount of phosphoSerine2056-DNA-PKcs in stable shCtr and shLRF U2OS cells treated with Bleomycin for 6, 12 and $24 \mathrm{~h}$ is shown. Average values of $n=3$ independent experiments are presented as mean values \pm s.d. Associated $P$ value calculated by Student's $t$-test analysis is indicated.

decreased in LRF knockdown compared with control cells (Fig. 4f). Endogenous DNA-PKcs autophosphorylation on serine 2056, a known correlate of DNA-PK activity ${ }^{32-36}$, was significantly reduced in LRF knockdown compared to control cells following treatment of cells with bleomycin (Fig. $4 \mathrm{~g}$ ). In keeping with these findings, in an in vitro assay using purified DNA-PKcs protein and extracts from wild-type and Lrf-deleted MEFs, we observed a substantially lower DNA-PKcs kinase activity in the absence of Lrf compared with controls (Fig. 5a).
LRF loss restores IR sensitivity in $p 53$ null cells. A characteristic feature of $p 53$ null cells is their resistance to $\mathrm{IR}^{37}$. This effect is reported to require normal DNA-PK function and loss of DNA-PKcs, Ku70 or Ku80 can restore the radiation sensitivity of $p 53$ null cells ${ }^{38}$. We therefore tested whether LRF loss, which is observed in advanced cancers ${ }^{15,17,18}$, could restore IR sensitivity in p53-deficient cells. Indeed, Lrf loss restored IR-induced apoptosis of p53 null MEFs (Fig. $5 \mathrm{~b}$ and Supplementary Fig. 4g). 
a

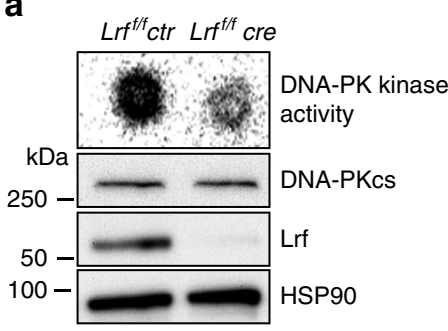

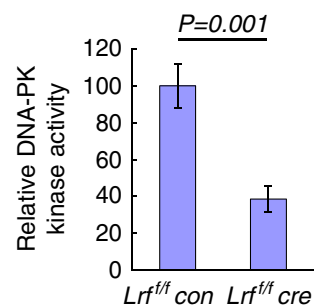

b

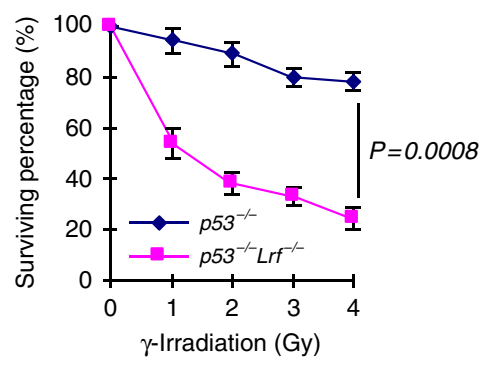

C

LRF proficient cells

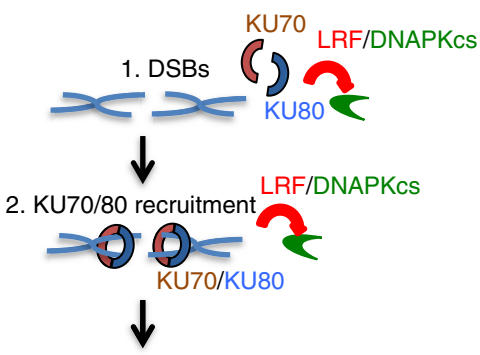

3. LRF DNA-PKcs KU70/80 complex (DNA-PK)

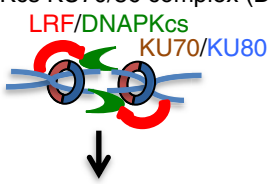

4. Stable and processive DNAPK complex

LRF/DNAPKcS

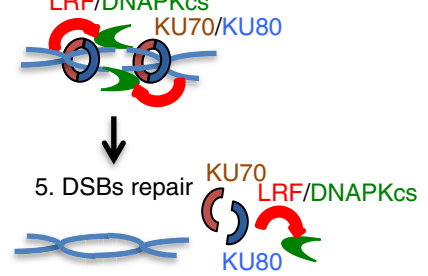

LRF deficient cells

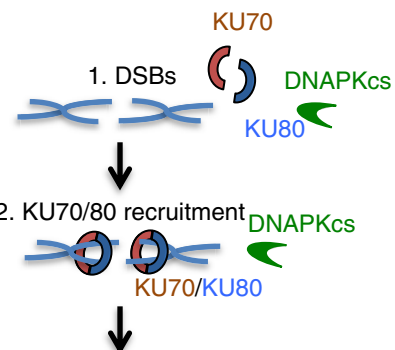

3. DNA-PKcs KU70/80 complex (DNA-PK)

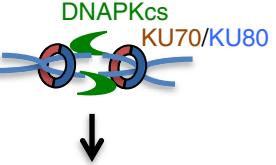

4. Premature DNA-PKcs release
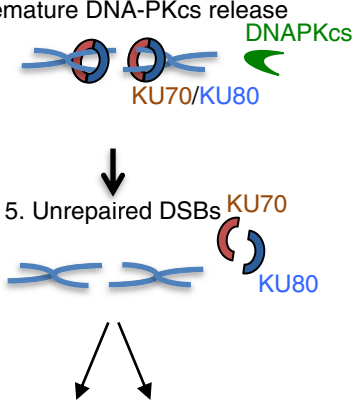

Restoration of therapy response to p53-null tumors through synthetic lethality

Figure 5 | LRF stabilizes the Ku/DNA-PKcs complex on DNA breaks. (a) DNA-PK kinase activity was assessed in total cell extracts from Arf ${ }^{-/-}$control

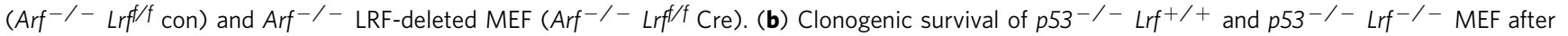
different doses of $\gamma$-radiation. (c) Schematic representation of LRF function in NHEJ DSBs repair pathway. Data are presented as mean \pm s.e.m. of three independent experiments. Associated $P$ value calculated by Student's $t$-test analysis is indicated.

\section{Discussion}

The human genome encodes $\sim 60$ POK family proteins ${ }^{16,19,39}$, containing an amino-terminal POZ domain and several carboxy-terminal $\mathrm{C} 2 \mathrm{H} 2$ Zinc finger domains. POK family proteins have been implicated in embryogenesis, the pathogenesis of cancer and other diseases primarily as transcriptional regulators of gene expression, although ZBTB1 has been recently shown to exert transcription-independent functions intriguingly associated with DNA repair ${ }^{40}$. Even though originally characterized as a proto-oncogene ${ }^{6}$, human $Z B T B 7 A$ is located at $19 \mathrm{p} 13.3$, a chromosomal region that is frequently lost in different types of human cancer, including prostate cancer $^{18,41,42}$. Interestingly, LRF has been recently characterized as a potent context-dependent tumour suppressor through the transcriptional repression of oncogenic pathways and glycolytic metabolism ${ }^{15-18}$. Here we identify LRF, a bonafide member of the POK family of proteins, as an important regulator of the DNA-PK complex required for the maintenance of genome integrity, which is a novel and unexpected function that LRF exerts independently of its transcriptional function. DNA-PKcs is the largest known protein kinase in the cell, which belongs to the phosphatidylinositol-3 (PI-3) kinase-related-kinase (PIKK) super-family based on primary structure. In current models, $\mathrm{Ku}$ association with DNA ends initiates a complex DNAPKcs-dependent signalling pathway through phosphorylation of downstream effectors responsible for DSB repair ${ }^{43}$. Importantly, this study unravels a novel and unexpected transcriptional independent function of POK family of proteins into the critical cellular processes of DNA-PK function and cNHEJ. Notably, BCL6 (B-cell lymphoma-6), a further member of the POK family and a key oncogenic driver in B-cell lymphoma ${ }^{44}$, has been demonstrated to physically bind $\mathrm{LRF}^{45}$ thus suggesting a possible role of BCL6 in the regulation of NHEJ. Importantly, LRF downregulation, caused by genetic loss or other mechanisms, 
has been recently reported to characterize specific subgroups of cancer patients ${ }^{15,17,18,46,47}$. As a novel component of the DNAPK complex and regulator of DNA-PK stability and activity (Fig. 5c), LRF represents an attractive biomarker with important therapeutic implications since its downregulation might serve to identify those tumours that are particularly dependent on NHEJ activity, such as for instance a subset of $p 53$-null cancers, towards therapeutic treatments based on genotoxic agents, radiation, or PARP inhibitors following the synthetic lethality paradigm.

\section{Methods}

Antibodies and reagents. Anti-Ku70 antibody (1 $\mu \mathrm{g}, \mathrm{BD}$ Biosciences \#611892), Anti-Ku80 antibody ( $1 \mu \mathrm{g}$, Cell Signaling Technology \#2753S), anti-DNA-PKcs antibody ( $1 \mu \mathrm{g}$, Bethyl Laboratories \#A300-517A), and anti-LRF antibody $(1 \mu \mathrm{g}$, Bethyl Laboratories \#A300-548A) were used for immunoprecipitation. Anti-Ku70 antibody (1:3,000, Cell Signaling D10A7 \#4588), Anti-Ku80 antibody (1:3,000, Cell Signaling Technology \#2753S), anti-DNA-PKcs antibody (1:3,000, Santa Cruz G4 \#sc-5282), and Anti-LRF antibody (1:1,000 13E9, Pandolfi lab), and anti phosphoS2056-DNA-PKcs (Cell Signaling Technology \#4215S), $\beta$-actin (1:5,000, Abcam \#ab8226), Rad51 (Scully lab; 1:1,000), Brcal (Scully lab; 1:1,000), LRF (13E9, Pandolfi lab; 1:1,000), Mre11 (Novus \#NB100-142; 1:1,000), Xrcc4 (Santa Cruz \#sc-8285; 1:1,000), anti- $\gamma-\mathrm{H} 2 \mathrm{AX}$ antibody (1:5,000 Cell Signaling, 20E3) were used for immunoblot. Anti- $\gamma-\mathrm{H} 2 \mathrm{AX}$ antibody (1:50 Cell Signaling, 20E3), anti-LRF antibody (1:50, 13E9 Pandolfi lab), anti-cleaved caspase-3 antibody (1:50, Cell Signaling, 5A1E) were used for immunohistochemistry. Bleomycin, Phleomycin, Mitomycin C, ICRF-193, and Camptothecin were purchased from Sigma. Purified DNA-PK complex was purchased from Promega.

Retrovirus transduction of mouse embryonic fibroblast. All animal procedures have been approved by the Beth Israel Deaconess Medical Center and Harvard Medical School institutional review board. Lrf ${ }^{+/}$, Lrflox/flox, $A r f^{-1-}$ and $p 53^{-1-}$ mice are previously described ${ }^{6,14,48,49} \cdot p 53^{-/-}$and $p 53^{-/-}$Lrf $^{-/-}$MEF were prepared from E13.5 mouse embryos obtained from the intercross of $p 53^{-1-}$

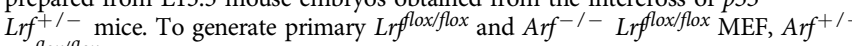
Lifflox/flox mice were intercrossed. MEFs were transduced with MSCV-PIG-Cre or empty control vector retrovirus for 2 days at passage 2 , and then selected with $2 \mu \mathrm{g} \mathrm{ml}^{-1}$ puromycin for 2 days before use in subsequent experiments.

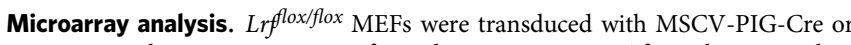
empty control vector retroviruses for 2 days at passage 2 . After selection with puromycin for 2 days, total RNAs were purified using the RNAeasy Mini Kit (Qiagen) and treated with RNase-free DNase set (Qiagen). RNAs from two independent experiments were labelled and hybridized using Affymetrix GeneChip HT Mouse Genome 430 arrays by the Beth Israel Deaconess Medical Center Genomics and Proteomics Center. Genes with normalized data values differing by a factor greater or less than 1.5 -fold were selected and further evaluated statistically.

Cell growth assay. Cells were seeded in 12 -well plates at a density of $10^{4} /$ well, then left to grow for 4 days. Cells were fixed by paraformaldehyde at each time point, and the cell number determined by crystal violet staining as described ${ }^{50}$.

Comet assay. DNA lesions were assessed using a single-cell gel electrophoretic comet assay kit (Trevigen). Cells were combined with low melting point agarose and pipetted onto a slide. The cells were lysed, then subject to electrophoresis at $20 \mathrm{~V}$ for $30 \mathrm{~min}$ in TBE buffer. Following electrophoresis, slides were washed, dehydrated and stained with SYBR Green I. Images were taken with a fluorescent microscope and scored by CometScore software (TriTek Corporation).

G-banding and telomere FISH of metaphase chromosome. Metaphase chromosome spreads were prepared from exponentially growing cells after treatment with demecolcine. For G-banding, the metaphase chromosomes were then treated with trypsin and stained with Giemsa according to standard procedures. Telomere FISH was performed using a Cy3-labelled peptide nucleic acid probe $\left(\mathrm{Cy} 3-(\mathrm{CCCTAA})_{3}\right)$ in metaphase chromosome spreads. Both the probe and the slides were heat denatured $\left(80^{\circ} \mathrm{C}\right.$ for $\left.5 \mathrm{~min}\right)$ and hybridized at $37^{\circ} \mathrm{C}$ for $2 \mathrm{~h}$. Slides were counterstained with DAPI. Images were captured using Zeiss microscope equipped with a CCD camera.

Protein complex purification and mass spectrometry. Procedures for LRFassociated protein complex purification have been described in detail previously ${ }^{51}$. Briefly, FLAG-HA tandem tagged human LRF was stably expressed in HeLa cells, then nuclear extracts were sequentially immunoprecipitated with anti-FLAG and anti-HA beads. The LRF binding proteins were separated using SDSpolyacrylamide gel electrophoresis (SDS-PAGE), and protein bands were identified by mass spectrometry.
Immunoblotting and immunoprecipitation. Cells were lysed in buffer $(50 \mathrm{mM}$ Tris, pH8.0, $150 \mathrm{mM} \mathrm{NaCl}$ and $0.5 \% \mathrm{NP}-40$ ). Protein concentrations of the lysates were measured by Bradford assay. The lysates were then resolved by SDS-PAGE and immunoblotted with the indicated antibodies. For immunoprecipitation, $1 \mathrm{mg}$ of cell lysate was incubated with the appropriate antibodies for $3-4 \mathrm{~h}$ at $4{ }^{\circ} \mathrm{C}$ followed by 1-h incubation with protein A beads (Santa Cruz). Immuno-complexes were washed with buffer $(20 \mathrm{mM}$ Tris, pH8.0, $100 \mathrm{mM} \mathrm{NaCl}, 1 \mathrm{mM}$ EDTA and $0.5 \%$ NP-40) before being resolved by SDS-PAGE and immunoblotted with the indicated antibodies. Uncropped scans of the most important blots are supplied as Supplementary Information.

Cell fractionation. $p 53^{-/-} \mathrm{Lrf}^{+/+}$and $p 53^{-/-} \mathrm{Lrf}^{-/-}$cells were incubated with $100 \mu \mathrm{M}$ phleomycin for 1 hour at $37^{\circ} \mathrm{C}$. The cell pellet was resuspended in buffer (150 mM NaCl, $50 \mathrm{mM}$ Hepes PH7.5, $1 \mathrm{mM}$ EDTA, 0.1\% Triton X-100, protease and phosphatase inhibitor) for $10 \mathrm{~min}$ on ice. Lysates were pelleted, and detergent extractable supernatant collected. The pellet was further extracted with buffer $\left(150 \mathrm{mM} \mathrm{NaCl}, 50 \mathrm{mM}\right.$ Hepes pH 7.5, $1 \mathrm{mM}$ EDTA, $200 \mu \mathrm{g} \mathrm{ml}^{-1}$ RNaseA, protease and phosphatase inhibitor) for $30 \mathrm{~min}$ at $25^{\circ} \mathrm{C}$. Then the RNase-resistant chromatin pellet was resuspended and sonicated in buffer $(150 \mathrm{mM} \mathrm{NaCl}, 25 \mathrm{mM}$ Tris $\mathrm{pH} 7.5,1 \% \mathrm{NP}-40,1 \%$ sodium deoxycholate, $0.1 \%$ SDS) before boiling in laemmli sampling buffer and immunoblot analysis.

DNA repair reporter assay. $2 \times 10^{5}$ ES cells stably integrated with NHEJ or HR reporter ${ }^{23,29}$ were transfected in suspension with $0.5 \mu \mathrm{g}$ pcDNA3 $\beta$-myc NLS-I-SceI or control pcDNA3 $\beta$ plasmid together with 20 pmol siRNA by using Lipofectamine 2000 (Invitrogen). In the U2OS HR reporter experiments, $1 \times 10^{5}$ cells were transiently transfected with $0.5 \mu \mathrm{g}$ pcDNA3 $\beta$-myc NLS-I-SceI or control pcDNA3 $\beta$ plasmid together with 20 pmol siRNA. In the U2OS NHEJ assays, $2 \times 10^{4}$ cells were first transfected with 30 pmol siRNA in Lipofectamine RNAiMAX on day zero, then received adeno-I-SceI (MOI of 5) $48 \mathrm{~h}$ later (with or without the DNA-PKcs inhibitor), with readout by FACS $72 \mathrm{~h}$ after adeno-I-SceI transduction. Transfection efficiency was measured simultaneously by parallel transfection with wild-type GFP expression plasmid, at an amount one-tenth of the I-SceI expression vector. GFP-positive cell frequencies were measured 3 days post transfection by flow cytometry in independent replicates and corrected for transfection efficiency.

siRNAs. Sequences of siRNAs are as follows: LRF-1: GAACCGACGACAAGG GCGU; LRF-2: GUAUAUAGAAUGCGGAUCA; LRF-3: CUACAGGCCUUU CGAGAUU. Brca1, Rad51, Xrcc4, hLRF are ON-TARGETplus siRNA - SMART pool (Dharmacon). siCtrl: siGENOME Non-Targeting siRNA \#2 (Dharmacon)

Laser-induced DNA damage and fluorescence data collection. Microirradiation with a pulsed $365 \mathrm{~nm}$ nitrogen laser (Spectra-Physics; $365 \mathrm{~nm}, 10 \mathrm{~Hz}$ pulse) was used to induce DSBs in the nuclei of U2OS cells. The laser system was directly coupled (Micropoint Ablation Laser System; Photonic Instruments, Inc.) to the epifluorescence path of the microscope (Axiovert 200M (Carl Zeiss MicroImaging, Inc.) for time-lapse imaging and focused through a Plan-Apochromat $\times 63 / \mathrm{NA}$ 1.40 oil immersion objective (Carl Zeiss MicroImaging, Inc.). Laser output was set at $75 \%$ of the maximum power, equivalent to the minimal dose required to induce detectable accumulation of YFP-DNA-PKcs in living cells. Time-lapse images were acquired with an AxioCam HRm (Carl Zeiss MicroImaging, Inc.). DNA-PKcs and Ku80 kinetics were calculated as previously described ${ }^{52}$ : fluorescence value of an undamaged spot in the same nuclei was subtracted from the fluorescence intensity of the laser-irradiated spot for every cell at each time point in order to eliminate the fluorescence background of the nucleus. Relative fluorescence intensity at each time point $(\mathrm{RF}(\mathrm{t}))$ was calculated as $\mathrm{RF}(\mathrm{t})=(\mathrm{INt}-\mathrm{INpreIR}) /(\mathrm{INmax}-\mathrm{INpreIR})$, where IN, fluorescence intensity; INpreIR, IN of the micro-irradiated area before laser damage; INmax, maximum IN in the micro-irradiated area.

DNA-damaging agents clonogenic survival assay. Four hundred cells were seeded in six-well plates $24 \mathrm{~h}$ before treatment with the indicated drugs. $\gamma$-radiation was supplied with a Cesium-137 source. After 10 days, colonies were stained with crystal violet and scored. A colony was defined as a cluster of more than $\sim 50$ cells. Cells without drug treatment were used as control. Survival ratio $=$ sample/ control $\times 100 \%$. Results were reported as mean \pm s.e.m. from three independent experiments.

DNA-PK kinase assay. DNA-PK kinase activity was measured using the SignaTECT DNA-Dependent Protein Kinase Assay System (Promega). Total cell lysates were extracted using a buffer containing $1 \% \mathrm{NP}-40,150 \mathrm{mM} \mathrm{NaCl}$ and $50 \mathrm{mM}$ Tris (pH8.0). Endogenous DNA of cell lysates was removed using Sepharose fast flow (GE Healthcare). For each reaction, $2 \mu \mathrm{g}$ of cell lysates were used, reactions were incubated at $30^{\circ} \mathrm{C}$ for $10 \mathrm{~min}$, and then the supernatant was spotted onto a SAM membrane. DNA-PK protein kinase activity was calculated as the incorporation of ${ }^{32} \mathrm{P}$ into the peptide using a phosphoimager. 
Immunofluorescence. Cells were seeded in 24-well plates containing round glass coverslips at the density of $2 \times 10^{4} /$ well. $24 \mathrm{~h}$ after plating, cells were fixed with paraformaldehyde, permeabilized in $0.1 \%$ Triton-X-100/phosphate buffered sulphate (PBS). Coverslips were then incubated with primary antibody diluted in $1 \%$ BSA/PBS for $1 \mathrm{~h}$. After washing, coverslips were incubated in secondary antibody diluted in $1 \%$ BSA/PBS for $1 \mathrm{~h}$. Coverslips were washed, stained with DAPI, mounted and analysed by confocal microscopy (Zeiss).

Flow cytometry. Cells were trypsinised, fixed with $4 \%$ formaldehyde and permeabilised with $90 \%$ methanol. Cells were then incubated with Alexa Fluor 647 conjugated $\gamma$-H2AX antibody (Cell Signaling Technology) in 0.5\% BSA /PBS for $1 \mathrm{~h}$ at room temperature. Cells were washed with $0.5 \%$ BSA /PBS then analysed by flow cytometry (LSR II, BD Biosciences). Data was analysed with FCS Express V3 software.

Chromatin immunoprecipitation assay. ChIP was performed using ES cells stably integrated with NHEJ reporter. $12 \mathrm{~h}$ after I-SceI expression, ES cells were trypsinised, cross-linked with $1 \%$ formaldehyde at $37^{\circ} \mathrm{C}$ for $10 \mathrm{~min}$, and stopped in $0.125 \mathrm{M}$ glycine. DNA was sonicated and incubated with indicated antibodies. Following reverse cross-linking, the associated DNA was extracted and detected by PCR using primers that locate $0.2 \mathrm{~kb}$ to I-SceI sites (p04.52, $5^{\prime}$-TGGTGAGCAAG GGCGAGGAGC- ${ }^{\prime}$; p04.53, $5^{\prime}$-TCGTGCTGCTTCATGTGGTCG-3') and $28 \mathrm{~kb}$ to I-SceI cutting sites (p07.52, 5'-TGTCATCATAGGCCCAATTTC-3'; p07.53, $5^{\prime}$-CCCAGTTTAAGGATGGTGGTT- $3^{\prime}$ ). Using Hotstart (Qiagen) enzyme, PCR condition: $95^{\circ} \mathrm{C} 15 \mathrm{~min}$ then $94^{\circ} \mathrm{C} 30 \mathrm{~s} ; 58^{\circ} \mathrm{C} 30 \mathrm{~s} ; 72^{\circ} \mathrm{C} 30$ s for 35 cycles. Samples were separated on a $2 \%$ agarose gel. ImageJ software was used to quantify the band intensity. The band intensity of PCR with IgG without I-SceI was set as 1 , and the relative ratio of PCR bands (using primers locate $0.2 \mathrm{~kb}$ to I-SceI site)/ PCR bands (using primers locate $28 \mathrm{~kb}$ to I-SceI site) are shown using data from three different experiments.

RT-qPCR Primers. Total RNA was extracted with TRIzol Reagents (Invitrogen) according to the provided protocol. $1 \mu \mathrm{g}$ total RNA was reversed transcribed with iScript cDNA Synthesis Kit (Bio-Rad). Real-time quantitative PCR was performed using diluted cDNA, SYBR Green JumpStart Taq ReadyMix (Sigma) and appropriate primers in StepOnePlus Real-Time PCR System (Applied Biosystems). Primers sequence is reported in Supplementary Table 4.

Statistical analysis. Results are expressed as mean \pm s.d or s.e.m as noted. Comparisons between groups were assessed using Student's $t$-test analysis. $P \leq 0.05$ was considered significant.

\section{References}

1. Negrini, S., Gorgoulis, V. G. \& Halazonetis, T. D. Genomic instability-an evolving hallmark of cancer. Nat. Rev. Mol. Cell. Biol. 11, 220-228 (2010).

2. Jackson, S. P. \& Bartek, J. The DNA-damage response in human biology and disease. Nature 461, 1071-1078 (2009).

3. Essers, J. et al. Homologous and non-homologous recombination differentially affect DNA damage repair in mice. EMBO J. 19, 1703-1710 (2000).

4. Boboila, C., Alt, F. W. \& Schwer, B. Classical and alternative end-joining pathways for repair of lymphocyte-specific and general DNA double-strand breaks. Adv. Immunol. 116, 1-49 (2012).

5. Lieber, M. R. The mechanism of double-strand DNA break repair by the nonhomologous DNA end-joining pathway. Annu. Rev. Biochem. 79, 181-211 (2010).

6. Maeda, T. et al. Role of the proto-oncogene Pokemon in cellular transformation and ARF repression. Nature 433, 278-285 (2005).

7. Pessler, F., Pendergrast, P. S. \& Hernandez, N. Purification and characterization of FBI-1, a cellular factor that binds to the human immunodeficiency virus type 1 inducer of short transcripts. Mol. Cell. Biol. 17, 3786-3798 (1997).

8. Kukita, A. et al. Osteoclast-derived zinc finger (OCZF) protein with POZ domain, a possible transcriptional repressor, is involved in osteoclastogenesis. Blood 94, 1987-1997 (1999).

9. Costoya, J. A. Functional analysis of the role of POK transcriptional repressors. Brief Funct. Genomic Proteomic 6, 8-18 (2007).

10. Kelly, K. F. \& Daniel, J. M. POZ for effect--POZ-ZF transcription factors in cancer and development. Trends. Cell. Biol. 16, 578-587 (2006).

11. Chen, Z. et al. Fusion between a novel Kruppel-like zinc finger gene and the retinoic acid receptor-alpha locus due to a variant $t(11 ; 17)$ translocation associated with acute promyelocytic leukaemia. EMBO. J. 12, 1161-1167 (1993).

12. Ye, B. H. et al. The BCL-6 proto-oncogene controls germinal-centre formation and Th2-type inflammation. Nat. Genet. 16, 161-170 (1997).

13. Maeda, T. et al. LRF is an essential downstream target of GATA1 in erythroid development and regulates BIM-dependent apoptosis. Dev. Cell 17, 527-540 (2009).
14. Maeda, T. et al. Regulation of B versus T lymphoid lineage fate decision by the proto-oncogene LRF. Science 316, 860-866 (2007).

15. Lunardi, A. et al. A co-clinical approach identifies mechanisms and potential therapies for androgen deprivation resistance in prostate cancer. Nat. Genet. 45, 747-755 (2013).

16. Lunardi, A., Guarnerio, J., Wang, G., Maeda, T. \& Pandolfi, P. P. Role of LRF/ Pokemon in lineage fate decisions. Blood 121, 2845-2853 (2013).

17. Wang, G. et al. Zbtb7a suppresses prostate cancer through repression of a Sox9-dependent pathway for cellular senescence bypass and tumor invasion. Nat. Genet. 45, 739-746 (2013).

18. Liu, X. S. et al. ZBTB7A acts as a tumor suppressor through the transcriptional repression of glycolysis. Genes Dev. 28, 1917-1928 (2014).

19. Lee, S. U. \& Maeda, T. POK/ZBTB proteins: an emerging family of proteins that regulate lymphoid development and function. Immunol. Rev. 247, 107-119 (2012).

20. Madison, B. B. et al. Cis elements of the villin gene control expression in restricted domains of the vertical (crypt) and horizontal (duodenum, cecum) axes of the intestine. J. Biol. Chem. 277, 33275-33283 (2002).

21. Kuhn, R., Schwenk, F., Aguet, M. \& Rajewsky, K. Inducible gene targeting in mice. Science 269, 1427-1429 (1995).

22. Redon, C. E. et al. Recent developments in the use of gamma-H2AX as a quantitative DNA double-strand break biomarker. Aging 3, 168-174 (2011).

23. Xie, A., Kwok, A. \& Scully, R. Role of mammalian Mre11 in classical and alternative nonhomologous end joining. Nat. Struct. Mol. Biol. 16, 814-818 (2009).

24. Lieber, M. R. \& Wilson, T. E. SnapShot: Nonhomologous DNA end joining (NHEJ). Cell 142, 496-496 e491 (2010).

25. Mladenov, E. \& Iliakis, G. Induction and repair of DNA double strand breaks: the increasing spectrum of non-homologous end joining pathways. Mutat. Res. 711, 61-72 (2011).

26. Yan, C. T. et al. IgH class switching and translocations use a robust nonclassical end-joining pathway. Nature 449, 478-482 (2007).

27. Gunn, A., Bennardo, N., Cheng, A. \& Stark, J. M. Correct end use during end joining of multiple chromosomal double strand breaks is influenced by repair protein RAD50, DNA-dependent protein kinase DNA-PKcs, and transcription context. J. Biol. Chem. 286, 42470-42482 (2011).

28. Bennardo, N., Cheng, A., Huang, N. \& Stark, J. M. Alternative-NHEJ is a mechanistically distinct pathway of mammalian chromosome break repair. PLoS Genet. 4, e1000110 (2008).

29. Puget, N., Knowlton, M. \& Scully, R. Molecular analysis of sister chromatid recombination in mammalian cells. DNA Repair (Amst). 4, 149-161 (2005).

30. Reynolds, P. et al. The dynamics of Ku70/80 and DNA-PKcs at DSBs induced by ionizing radiation is dependent on the complexity of damage. Nucleic Acids Res. 40, 10821-10831 (2012).

31. Suwa, A. et al. DNA-dependent protein kinase (Ku protein-p350 complex) assembles on double-stranded DNA. Proc. Natl Acad. Sci. USA 91, 6904-6908 (1994).

32. Chen, B. P. et al. Cell cycle dependence of DNA-dependent protein kinase phosphorylation in response to DNA double strand breaks. J. Biol. Chem. 280, 14709-14715 (2005).

33. Chan, D. W. et al. Autophosphorylation of the DNA-dependent protein kinase catalytic subunit is required for rejoining of DNA double-strand breaks. Genes Dev. 16, 2333-2338 (2002).

34. Cui, X. et al. Autophosphorylation of DNA-dependent protein kinase regulates DNA end processing and may also alter double-strand break repair pathway choice. Mol. Cell Biol. 25, 10842-10852 (2005).

35. Merkle, D. et al. The DNA-dependent protein kinase interacts with DNA to form a protein-DNA complex that is disrupted by phosphorylation. Biochemistry 41, 12706-12714 (2002).

36. Block, W. D. et al. Autophosphorylation-dependent remodeling of the DNA-dependent protein kinase catalytic subunit regulates ligation of DNA ends. Nucleic Acids Res. 32, 4351-4357 (2004).

37. Gudkov, A. V. \& Komarova, E. A. The role of $\mathrm{p} 53$ in determining sensitivity to radiotherapy. Nat. Rev. Cancer. 3, 117-129 (2003).

38. Gurley, K. E., Moser, R., Gu, Y., Hasty, P. \& Kemp, C. J. DNA-PK suppresses a p53-independent apoptotic response to DNA damage. EMBO Rep. 10, 87-93 (2009).

39. van Roy, F. M. \& McCrea, P. D. A role for Kaiso-p120ctn complexes in cancer? Nat. Rev. Cancer. 5, 956-964 (2005).

40. Kim, H. et al. Transcriptional repressor ZBTB1 promotes chromatin remodeling and translesion DNA synthesis. Mol. Cell 54, 107-118 (2014)

41. Sobottka, S. B. et al. Frequent loss of heterozygosity at the 19p13.3 locus without LKB1/STK11 mutations in human carcinoma metastases to the brain. J. Neurooncol. 49, 187-195 (2000).

42. Yanaihara, N., Okamoto, A. \& Matsufuji, S. A commonly deleted region in ovarian cancer on chromosome 19p13.3, not including the OAZ1 gene. Int. J. Oncol. 23, 567-575 (2003). 
43. Collis, S. J., DeWeese, T. L., Jeggo, P. A. \& Parker, A. R. The life and death of DNA-PK. Oncogene 24, 949-961 (2005).

44. Ye, B. H. et al. Alterations of a zinc finger-encoding gene, BCL-6, in diffuse large-cell lymphoma. Science 262, 747-750 (1993).

45. Davies, J. M. et al. Novel BTB/POZ domain zinc-finger protein, LRF, is a potential target of the LAZ-3/BCL-6 oncogene. Oncogene 18, 365-375 (1999).

46. Guarnerio, J. et al. A genetic platform to model sarcomagenesis from primary adult mesenchymal stem cells. Cancer Discov. 5, 396-409 (2015).

47. Bohn, O. et al. Utility of LRF/Pokemon and NOTCH1 protein expression in the distinction between nodular lymphocyte-predominant Hodgkin lymphoma and classical Hodgkin lymphoma. Int. J. Surg. Pathol. 22, 6-11 (2014).

48. Kamijo, T. et al. Tumor suppression at the mouse INK4a locus mediated by the alternative reading frame product p19ARF. Cell 91, 649-659 (1997).

49. Jacks, T. et al. Tumor spectrum analysis in p53-mutant mice. Curr. Biol. 4, 1-7 (1994).

50. Carnero, A., Hudson, J. D., Price, C. M. \& Beach, D. H. p16INK4A and p19ARF act in overlapping pathways in cellular immortalization. Nat. Cell. Biol. 2, 148-155 (2000).

51. Ogawa, H., Ishiguro, K., Gaubatz, S., Livingston, D. M. \& Nakatani, Y. A complex with chromatin modifiers that occupies E2F- and Myc-responsive genes in G0 cells. Science 296, 1132-1136 (2002).

52. Shao, Z. et al. Persistently bound $\mathrm{Ku}$ at DNA ends attenuates DNA end resection and homologous recombination. DNA Repair (Amst). 11, 310-316 (2012).

\section{Acknowledgements}

We are particularly grateful to Dr Fred Alt for his insightful comments and suggestions. We thank current and former members of the Pandolfi laboratory for experimental support and advice. We thank Dr Yufei Xu for assistance in protein complex purification, Dr John Asara for mass-spectrometric analysis, Ms. Shih-Ya Wang for assisting with the laser experiments and data collection, Dr Hong Wang for critical discussion. This work was supported in part by from the National Cancer Institute grants to P.P.P.; by NCI and NIGMS grants to R.S.; by The Giovanni Armenise-Harvard Foundation Career
Development Award to A.L.; by a postdoctoral training fellowship from Department of Defense to X.-S.L.; and by a postdoctoral training fellowship from the Susan G. Komen Foundation to E.R.

\section{Author contributions}

X.S.L., A.L., R.S. and P.P.P. designed experiments. X.S.L., A.L., G.C., A.J.D., E.R., Y.G. and A.R. performed experiments. X.S.L., A.L., J.V.S., A.J.D., R.S., P.P.P. analysed data. G.W., R.M.H., A.X. and J.V.S. helped to interpret data. X.S.L., R.S., A.L. and P.P.P. wrote the manuscript.

\section{Additional information}

Accession codes: The microarray data has been deposited to the Gene Expression Omnibus at the National Center for Biotechnology Information under the accession code GSE70780.

Supplementary Information accompanies this paper at http://www.nature.com/ naturecommunications

Competing financial interests: The authors declare no competing financial interests.

Reprints and permission information is available online at http://npg.nature.com/ reprintsandpermissions/

How to cite this article: Liu, X. S. et al. LRF maintains genome integrity by regulating the non-homologous end joining pathway of DNA repair. Nat. Commun. 6:8325 doi: $10.1038 /$ ncomms9325 (2015).

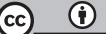

This work is licensed under a Creative Commons Attribution 4.0 International License. The images or other third party material in this article are included in the article's Creative Commons license, unless indicated otherwise in the credit line; if the material is not included under the Creative Commons license, users will need to obtain permission from the license holder to reproduce the material. To view a copy of this license, visit http://creativecommons.org/licenses/by/4.0/ 